\title{
Swirl flow focusing: A novel procedure for the massive production of monodisperse microbubbles
}

\author{
Miguel A. Herrada and Alfonso M. Gañán-Calvo \\ Escuela Superior de Ingenieros, Universidad de Sevilla, Camino de los Descubrimientos s/n, \\ Sevilla 41092, Spain
}

(Received 20 November 2008; accepted 26 March 2009; published online 30 April 2009)

\begin{abstract}
A volume-of-fluid numerical method is used to predict the dynamics of microbubble formation in an axisymmetric flow-focusing microfluidic device for a gas-liquid system. Numerical results show that, in all the cases analyzed, the introduction of swirl into the focusing liquid stabilizes the tapering gas-liquid meniscus from which a steady gas ligament issues. Consequently, a drastic reduction in the size of bubbles generated by the device is achieved under similar gas and liquid flow rates. (C) 2009 American Institute of Physics. [DOI: 10.1063/1.3123533]
\end{abstract}

\section{INTRODUCTION}

Research on the production and dynamics of microbubbles has recently undergone a sudden boost owing to its biomedical applications. According to Wells, ${ }^{1}$ one of the major milestones in medicine was the invention of ultrasound imaging. The use of microbubbles has revolutionized this field owing to their dramatic imaging contrast enhancement. ${ }^{2} \mathrm{~A}$ further application under steady development is drug or gene delivery by microbubbles activated by ultrasound. ${ }^{3,4}$ Additional possibilities such as blood oxygenation $^{5,6}$ and direct injection or perfusion of different therapeutic gases are being pursued. Depending on the particular application, the long term stability of these microbubbles may become a key requirement. It is currently addressed by the use of lipidic shells ${ }^{7}$ and the combined use of perfluorocarbons. ${ }^{5,6}$ Nonetheless, from the fundamental physical side, one finds two basic requirements everywhere in the biomedical literature for these applications: ${ }^{8}$ (i) sufficiently small size, to avoid embolisms, cloth generation, and to retard macrophage activation and (ii) size homogeneity (monodispersity).

Microbubbles can be produced in large quantities by ultrasound irradiation onto a liquid bulk with a free surface, but this process generates major size disparity and thus demands further operations to sort out the target sizes (see Ref. 9 and references therein). Recently, electrohydrodynamic jetting has also been proposed for the generation of microbubbles ${ }^{10,11}$ resulting in less polydisperse populations of smaller bubbles, but it requires the use of high viscosity liquids. Microfluidics has also been demonstrated to be an attractive means to mass-produce perfectly monodisperse microbubbles with selectable size in a wide variety of liquids. ${ }^{712-14}$ The different microfluidic setups can be classified into planar ${ }^{13,15}$ or axisymmetric $\mathrm{T}$ junctions ${ }^{16,17}$ or flowfocusing schemes. ${ }^{12,18}$ Flow focusing was initially proposed $^{19}$ as a simple means to disperse a given fluid phase into another immiscible phase (carrier phase) in the form of corpuscles (droplets) with a small size compared to any other scale of the generating device. The process resorts to the formation of a steady liquid microjet ${ }^{20}$ focused by a coflow- ing stream of the carrier phase forced through a small aperture (large compared to the droplet size) and yields remarkable droplet size control: it can be selectable and homogeneous, at least over a reasonably wide range of conditions. Furthermore, with the same idea of seeking for stable tapering menisci focused by coflowing streams, flow focusing was proposed for the generation of perfectly monodisperse microbubbles as well, ${ }^{12}$ but in this case no steady capillary jet was observed, at least in the parameter range explored: the bubbles seemed to be produced just at the apex of the steady tapering meniscus formed by the focusing liquid stream.

The size of the microbubbles was subsequently shown ${ }^{21}$ to follow a simple scaling law as

$$
R_{b} / R=1.1\left(Q_{g} / Q_{l}\right)^{2 / 5},
$$

where $R_{b}, R, Q_{g}$, and $Q_{l}$ are the bubble and the orifice radii and the gas and liquid flow rates, respectively. This incomplete similarity law ${ }^{22}$ of the first kind was obtained from the assumption that local and convective liquid accelerations at the region where the bubble is formed were, as an average, of the same order throughout the cycle. Garstecki et al. ${ }^{23}$ successfully proposed other mechanisms of monodisperse bubble formation when viscous forces dominate and explored other regimes where the system exhibited complex dynamics. ${ }^{24}$ Clearly though, the sizes of the bubbles formed were in general significantly smaller when the focused tapering meniscus was steady than in the regimes where it oscillated. Under oscillation, the bubble size is comparable to the scales of the device (e.g., the aperture or the distance from the feeding channel to the aperture) as observed in other flow-focusing cases; $;^{25}$ in the steady regime, the bubble size is determined by the gas and liquid flow rates.

The width of the parameter range where very small bubbles are formed was explored by Gañán-Calvo and Gordillo, ${ }^{12}$ and Garstecki and co-workers ${ }^{13,14}$ showing that, when the gas flow rate was decreased below about $10^{-2}$ times the liquid flow rate, the tapering meniscus oscillated and the bubble size suddenly increased dramatically. In fact, the stabilization of the tapering meniscus was early seen to be the key factor to achieve extremely small sizes in flow focusing. 


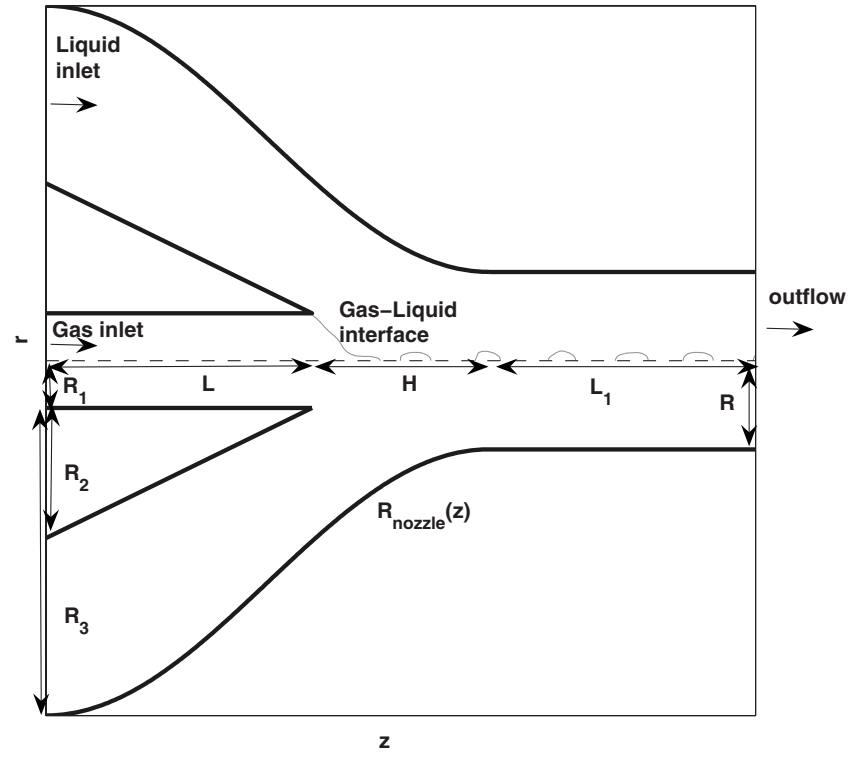

FIG. 1. Simulated boundary geometry and fluid flow domains.

In the present work, we propose a simple and remarkably effective way to enhance the stability of the tapering meniscus down to relative gas-to-liquid flow rates as small as 1 part in 10 000: the upstream introduction of a coaxial swirl in the coflowing liquid stream. Accordingly, bubble sizes below $5 \%$ of the exit orifice or channel size can be obtained. In this preliminary work we shall describe the first numerical results of our novel proposed microbubbling method, showing the chief stabilizing role of the swirl. Following the procedures established by Gañán-Calvo and co-workers, linear stability analysis ${ }^{26}$ and, most importantly, full numerical Navier-Stokes simulation by the volume-of-fluid (VoF) method $^{27}$ are here used to provide physical insight into the dynamics, explore the parameter space, and determine the stability limits of the system. In Ref. 27 the numerical VoF method showed remarkable accuracy in describing and predicting the most intricate details of interest in moderate-tohigh Reynolds flows with free surface. Again, this work is naturally not intended to exhaust the subject just discovered, but on the contrary to foster further investigation form the very promising results yielded by our numerical exploration. In this regard, a systematic experimental study by our group is now under way.

\section{GOVERNING EQUATIONS AND BOUNDARY CONDITIONS}

The axisymmetric flow-focusing device and the computational domain used in this work are sketched in Fig. 1. A constant gas flow rate $Q_{g}$, flowing through a capillary tube (outer radius $R_{2}$, inner radius $R_{1}$ ), is discharged coaxially in a coflowing liquid stream forced through a coaxial converging tube of final (discharge) radius $R$ located at a downstream distance $H$ from the needle edge. The gas stream is then drawn by a constant flow rate $Q_{l}$ of the focusing swirling liquid stream flowing through the gap formed between the needle and the coaxial tube, whose radius upstream of the discharge of the capillary tube is $R_{3}$. The liquid is forced into the downstream tube of smaller radius with the help of a converging nozzle:

$$
\begin{aligned}
& R_{\text {nozzle }}(z)=R_{3}\left[1+\alpha \cos \left(\frac{\pi z}{H+L}\right)-\alpha\right], \\
& \alpha=\frac{R_{3}-R}{2 R_{3}}, \quad 0 \leq z \leq L+H .
\end{aligned}
$$

Due to the relatively small pressure variations used, for the purposes of this work and for simplicity of formulation the gas is assumed to be incompressible. Therefore, incompressible, axisymmetric, and unsteady Navier-Stokes equations in cylindrical $(z, r, \phi)$ coordinates are used to describe the time evolution of both fluids, where $\mathbf{u}=(w, u, v)$ and $p$ are the velocity and pressure fields, respectively.

Figure 1 also shows the boundary conditions: (a) at the gas inlet, $z=0$, a Hagen-Poiseuille flow, $W_{g}(r)=W_{1}[1$ $\left.-\left(r / R_{1}\right)^{2}\right]$ and $V_{g}=0$, is specified; (b) at the liquid inlet, $z$ $=0, \quad R_{2}<r<R_{3}$, we assume a parabolic profile, $W_{l}(r)$ $=W_{2}\left(R_{3}-r\right)\left(r-R_{2}\right) /\left(R_{3}-R_{2}\right)^{2}$, for the axial velocity, while a solid-body profile, $V_{l}(r)=\Omega r$, for the azimuthal velocity is considered; (c) $\mathbf{u}=0$ on all solid walls; (d) at the axis $r=0$ a symmetry condition is applied; and (e) at the pipe exit, $z$ $=z_{\text {out }}=L+H+L_{1}$, outflow conditions $\partial \mathbf{u} / \partial z=0$ are considered. Note that the corresponding gas and liquid flow rates can be derived from the inlet velocity field,

$$
Q_{g}=\int_{0}^{R_{1}} 2 \pi r W_{g}(r) d r, \quad Q_{l}=\int_{R_{2}}^{R_{3}} 2 \pi r W_{l}(r) d r .
$$

Parametric studies of the dimensionless variables involved are carried out next. The velocity field $\mathbf{u}$, lengths, time $t$, and pressure $p$ are scaled with the mean axial liquid velocity at the pipe $W=Q_{l} /\left(\pi R^{2}\right)$, the discharge tube radius $R, R / W$, and $\rho_{l} W^{2}$, respectively, where $\rho_{l}$ is the density of the focusing liquid. In this preliminary work, a single geometry is considered, characterized by the following aspect ratios: $R_{1} / R=0.533, R_{2} / R=2, R_{3} / R=4, H / R=2, L / R=3$, and $L_{1} / R=8$. We have chosen a gas-liquid combination where

$$
\frac{\rho_{l}}{\rho_{g}}=833.33, \quad \frac{\mu_{l}}{\mu_{g}}=55.55,
$$

in which $\rho_{l}\left(\rho_{g}\right)$ and $\mu_{l}\left(\mu_{g}\right)$ are the density and viscosity of the liquid (gas). This choice corresponds to an air stream drawn by a coflowing water stream. The problem is governed by a set of four nondimensional numbers as follows.

- The Reynolds and Weber numbers defined as

$$
\begin{gathered}
\mathrm{Re}=\frac{\rho_{l} W R}{\mu_{l}}, \\
\mathrm{We}=\frac{\rho_{l} W^{2} R}{\sigma},
\end{gathered}
$$

$\sigma$ being the surface tension between the two phases.

- The flow rate ratio $Q=Q_{g} / Q_{l}$.

- The swirl parameter, $S$, defined as 


$$
S=\frac{\Omega R_{3}^{2}}{W R},
$$

where $\Omega R_{3}^{2} / R$ is the characteristic azimuthal velocity of the liquid at the nozzle.

For a given value of Re and We wish to analyze the formation of monodisperse bubbles and the dependence of the flow on the quotient $Q$ and the swirl parameter $S$. Under different liquid flow conditions, we will demonstrate that the smaller monodisperse bubbles are always obtained when the focusing liquid is swirled $(S>0)$. We will also show that the $Q$ range where monodisperse bubbles are possible expands as the swirl intensity of the focusing liquid increases. Naturally, the swirl effect depends on its persistency and its amplification when the liquid stream is forced through the constriction and focused into the near-axis region. In turn, focusing can only be observed when quasistagnant situations (low Reynolds number, leading to bubble plug flow) are excluded. Therefore, only moderate-to-high Reynolds numbers are considered in this work. In particular, we consider here in detail five different conditions for the focusing liquid:

- Case 1: $\mathrm{Re}=300, \mathrm{We}=16.66$.

- Case 2: $\mathrm{Re}=1200, \mathrm{We}=266.66$.

- Case 3: $\mathrm{Re}=1800, \mathrm{We}=600$.

- Case 4: $\mathrm{Re}=300, \mathrm{We}=33.33$.

- Case 5: $\mathrm{Re}=1200, \mathrm{We}=533.33$.

For fixed values of $R, \rho_{l}, \mu_{l}$, and $\sigma$, cases 1-3 explore an increase in the liquid flow rate $Q_{l}$. Cases 4 and 5 represent the same situation as cases 1 and 2 assuming half the original value of the surface tension $\sigma$. Each case will be explored for a variety of $Q$ and $S$ values.

\section{NUMERICAL PROCEDURE}

In order to predict the interface geometry during the time solution, several techniques have been used, falling into one of three categories. These are (i) interface tracking methods, including a moving mesh, ${ }^{28}$ (ii) front tracking and particle tracking schemes, ${ }^{29}$ and (iii) interface capturing methods, including VoF (Refs. 30 and 31) and level set techniques. ${ }^{32} \mathrm{We}$ chose a VoF method consisting of two parts: an interface reconstruction algorithm to approximate the interface from the set of volume fractions and a VoF transport algorithm to determine the volume fraction at the new time level from the velocity field and the reconstructed front. The basic method is robust and flexible and is based on widely used VoF schemes. ${ }^{33-36}$

We used the commercial solver FLUENT v 6.2 (laminar unsteady) to resolve the discretized mass continuity, momentum conservation, and liquid volume fraction equations in the mesh depicted in Fig. 2, generated by the commercial code GAMBIT in FLUENT v 6.2. The cells are quadrangles, mostly square. The basic mesh should be sufficiently refined to capture, in the absence of the gas, the strong velocity gradients experienced by the liquid flow at the nozzle region. In the grid shown in Fig. 2 the radial and axial lengths of the quadrilateral cells are $\left(\Delta_{z}\right)_{\text {square }}=\left(\Delta_{r}\right)_{\mathrm{square}}=0.0216$. Several

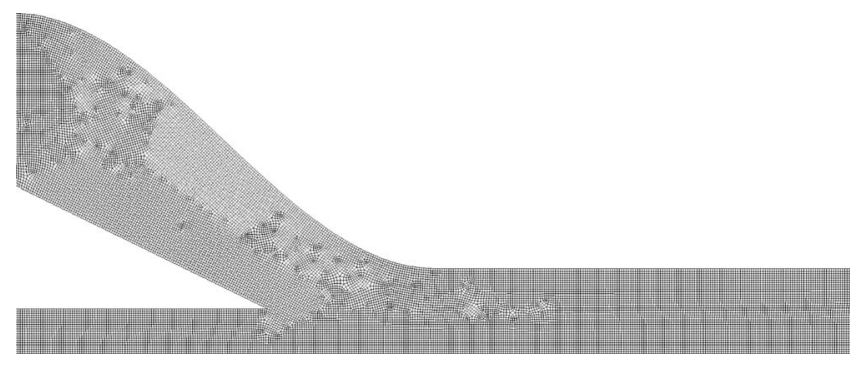

FIG. 2. Basic grid of the domain under study.

numerical tests with smaller size mesh cells have shown that this accuracy level is comfortably sufficient to describe the liquid flow pattern for the three cases considered $(\mathrm{Re}=300$, 1200 , and 1800) and for the range of values of the swirl parameter used $(0 \leq S \leq 1.2)$. All results presented here were initially computed on that mesh. To avoid numerical diffusion of the interface when a gas stream is supplied, a refined mesh with quadrilateral cells $\left(\Delta_{z}\right)_{\text {square }}=\left(\Delta_{r}\right)_{\text {square }}=0.0054$ was used in the interfacial region.

Tracking the interface between the phases is accomplished by solving a continuity equation for the volume fraction of one of the phases using an explicit time-marching scheme. The rest of the equations are solved implicitly. The time steps selected were fixed and sufficiently small to ensure that the global Courant number based on the mesh cell size, the mean velocity in the cell, and the time step was always smaller than 1 . Regarding the spatial discretization of the equations, the third-order modified monotone upstreamcentered schemes for conservation laws (MUSCL) scheme ${ }^{37}$ is used to obtain the face fluxes whenever a cell is completely immersed in a single phase. When the cell is near the interface, the georeconstruction algorithm is used. The pressure corrections are computed with the body forces weighted scheme, and the pressure-velocity coupling in a segregated solver is treated with the pressure-implicit with splitting of operators (PISO) method. ${ }^{38}$

\section{NUMERICAL RESULTS}

\section{A. Nonswirling case $S=0$}

When there is no swirl, our numerical simulations show that the bubble generation in the flow-focusing devices is quite similar for the five cases considered: in all cases and for any value of the flow rate quotient $Q$, the bubbles are generated from a nonsteady gas-liquid meniscus in a bubbling regime. To characterize the dripping frequency and size of the emitted bubbles, the bubble chord radius, defined as the radius of the instantaneous section of the bubble at the outlet plane, $R_{\text {bubble }}(t)$, is computed at each time step by integrating radially the gas volume fraction $\alpha$ at the outlet of the device, $z=z_{\text {out }}$,

$$
R_{\text {bubble }}(t)=\sqrt{2 \int_{0}^{1} \alpha\left(t, z_{\mathrm{out}}, r\right) r d r} .
$$

Figure 3 show this quantity as a function of time for case 1 and four different values of $Q$. Note that when a bubble is crossing the outlet, the instantaneous chord radius $R_{\text {bubble }}$ is 

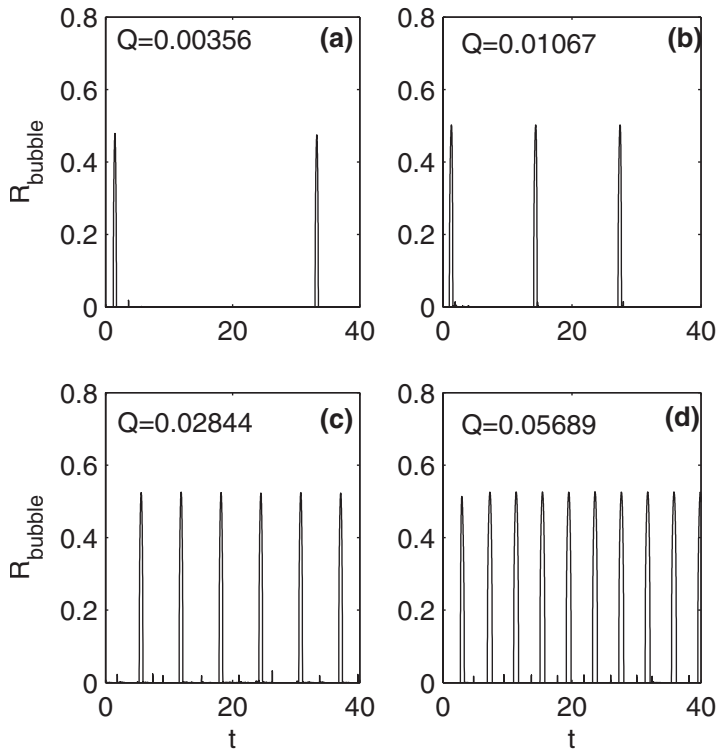

FIG. 3. Time dependence of the instantaneous bubble chord radius at the outlet, $R_{\text {bubble }}$, for case 1 and four different values of $Q$.

positive, while $R_{\text {bubble }}=0$ otherwise. Each local maximum, $R_{\text {bubble, }}^{\max }$, provided an estimate of the bubble radius at the outlet. In fact, if the bubble is completely spherical, the local maximum of $R_{\text {bubble }}$ gives the exact radius of the bubble. The figure shows that for the four cases depicted, mostly monodisperse bubbles are generated with a constant frequency depending on the flow quotient $Q$ : the larger $Q$, the smaller the frequency. Note also that the size of the bubbles increases slightly with $Q$. Figures 4(a)-4(f) show an example of the bubble generation for case 1 and $Q=0.01066$, going through the stages of meniscus growth and bubble emission. The shape of the gas-liquid interface is computed in the figure as the isolevel of the gas volume fraction $\alpha=0.999$, obtained with the VoF method. Note that, during the pinching process, a small satellite bubble is generated. For this particular case, the satellite bubble is absorbed by the main bubble downstream in the tube.

The above results for case 1 show that there is a relatively large $Q$ range where monodisperse bubbles can be
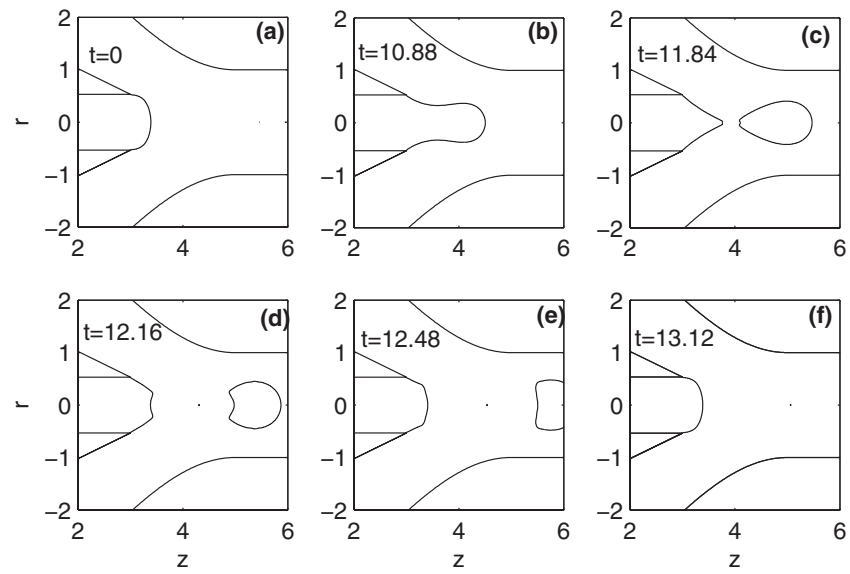

FIG. 4. A sequence of snapshots showing bubble generation for case 1 and $Q=0.01067$. In this case, the capillary meniscus oscillates.
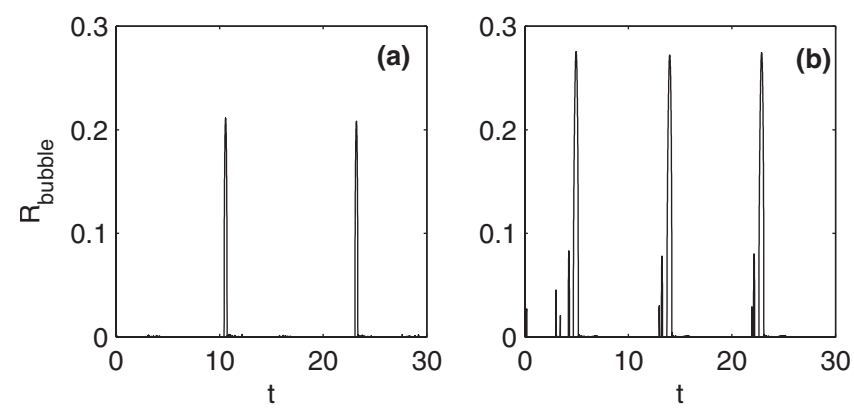

FIG. 5. Time dependence of the instantaneous bubble chord radius at the

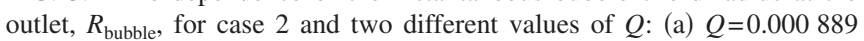
and (b) $Q=0.003556$.

generated. The situation is different for cases 2 and 3 where monodisperse bubbles are obtained only for a very narrow $Q$ range. In such cases, when $Q$ is increased, the size and number of satellite bubbles rise sharply. To illustrate this, we have plotted in Fig. $5 R_{\text {bubble }}$ as a function of time for case 2 and two different values of $Q$. Observe that for $Q$ $=0.003556$ satellite bubbles are observed interspersed between the main bubbles. A comparison between the maximum values of $R_{\text {bubble }}$ in Figs. 3 and 5 also shows that in case 2 the main bubbles are smaller than in case 1 . The size reduction in the main bubble is even greater in case 3 . This can be observed in Fig. 6, showing $R_{\text {bubble }}$ as a function of time for case 3 and two different values of $Q$. This figure also shows that, for $Q=0.002370$, the bubbling process becomes quite irregular. The results for cases 1-3 show that, for a fixed geometry and fixed gas-liquid properties, an increase in the liquid flow rate $Q_{l}$ yields a reduction in the bubble size produced but also contracts the $Q$ range where monodisperse bubbles can be generated.

Let us now focus attention on cases 4 and 5, similar to 1 and 2, respectively, but with halved surface tension. Figure 7 shows $R_{\text {bubble }}$ as a function of time for case 4 and two different values of $Q$. If we compare the results of this figure with Fig. 3 (case 1) it is readily observed that, for a given value of $Q$, the reduction in the surface force leads to a reduction in the bubble size. The same situation is observed for other liquid configurations. For example, the results depicted in Fig. 8 for case 5 clearly show that the bubble radii are smaller (for the same value of $Q$ ) than in case 2 (Fig. 5).
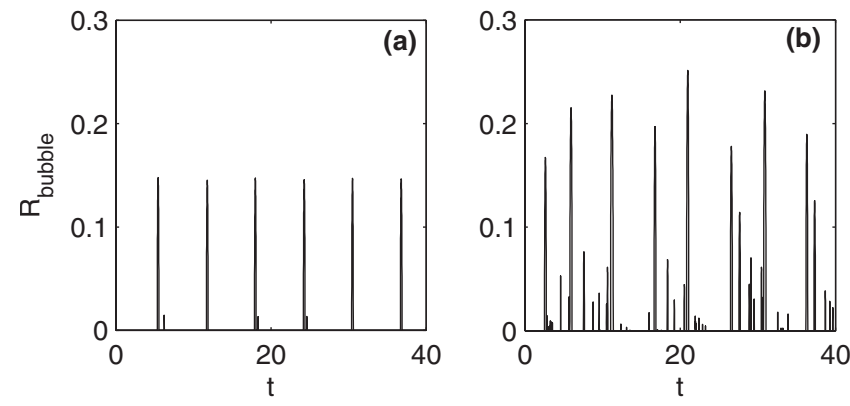

FIG. 6. Time dependence of the instantaneous bubble chord radius at the

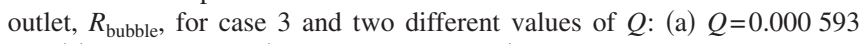
and (b) $Q=0.002370$ (polydisperse breakup). 
(a)

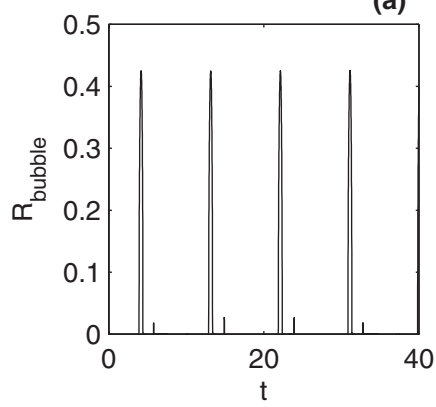

(b)

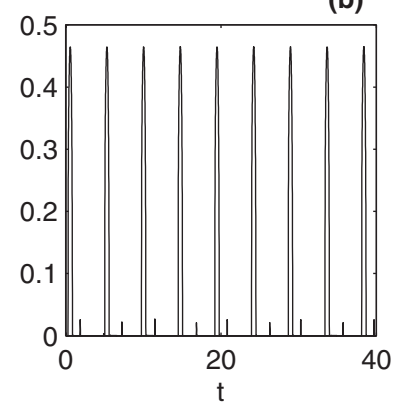

FIG. 7. Time dependence of the instantaneous bubble chord radius at the outlet, $R_{\text {bubble }}$, for case 4 and two different values of $Q$ : (a) $Q=0.001067$ and (b) $Q=0.002844$.

\section{B. Swirling case $S>0$}

Our numerical simulations show that, upon introduction of swirl, the size of the bubbles generated by the flowfocusing device can be reduced drastically with respect to the nonswirling case for all the five cases analyzed, and also that the $Q$ range where monodisperse bubbles are obtained is expanded. This is caused by the centrifugal forces associated with the swirl: they help, in a certain range of $S$, stabilize the gas-liquid meniscus. To illustrate this statement, Fig. 9 shows the generation of monodisperse bubbles for case $1, Q$ $=0.01067$ and $S=1.2$. Contrarily to the nonswirl results in the same case (Fig. 4), the bubbles are emitted from a short steady gas-liquid meniscus, and their sizes are much smaller. The effect of the swirl parameter $S$ on the bubble generation is shown quantitatively in Fig. 10 where $R_{\text {bubble }}$ as a function of time is depicted for case $1, Q=0.1067$, and three values of $S$. It can be seen that the bubble size reduction owing to swirl leads to a rise in the bubble generation frequency for a given value of $Q$. We next carried out an extensive study to find the parameter region where monodisperse bubbles are obtained. In this parameter region we characterized the size of the bubbles as a function of $Q$ and $S$. The exact volume of each bubble crossing the outlet is computed using the function

$$
Q_{\text {gas }}(t)=\int_{0}^{1} \alpha(t, z=13, r) w(t, z=13, r) 2 \pi r d r .
$$

This quantity provides the instantaneous gas flow rate at the outlet, $Q_{\text {gas }}$, which is zero when there are no bubbles cross-

(a)

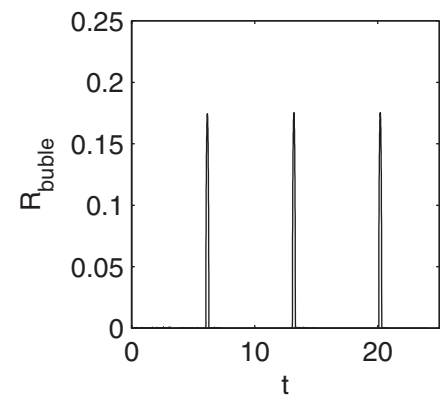

(b)

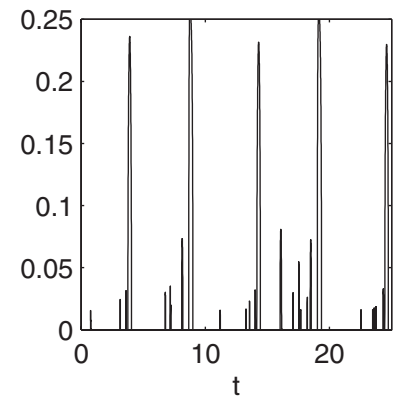

FIG. 8. Time dependence of the instantaneous bubble chord radius at the

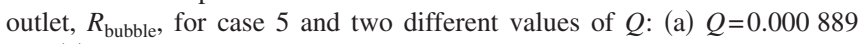
and (b) $Q=0.003556$.
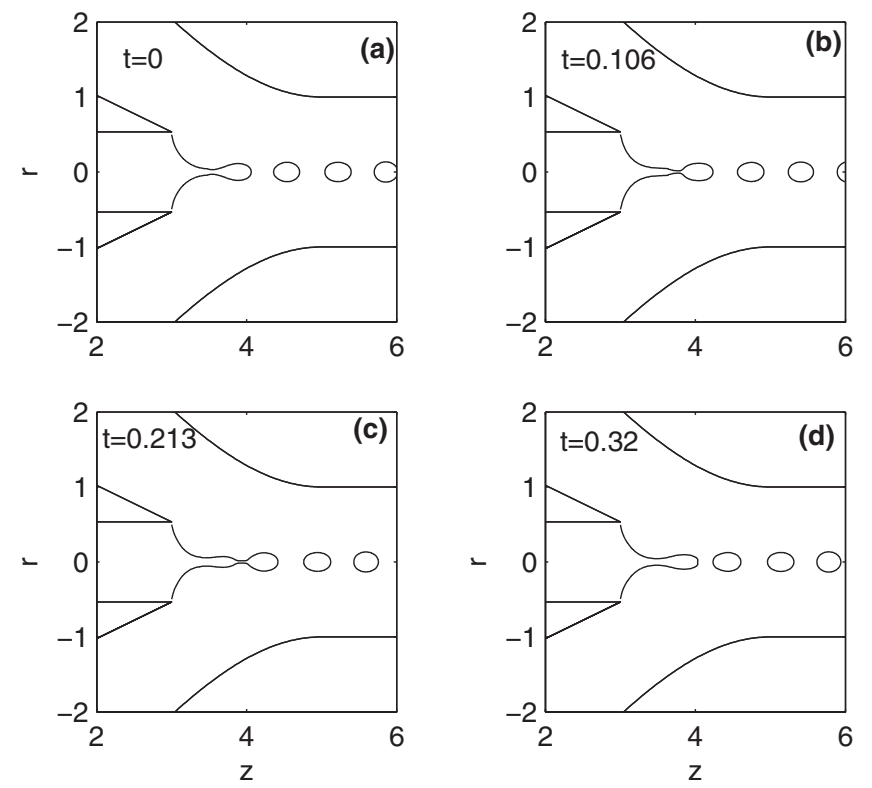

FIG. 9. A sequence of snapshots showing bubble formation for case $1, Q$ $=0.01067$ and $S=1.2$. In this case, the liquid meniscus from which the microbubbles issue is clearly stable (it does not oscillate).

ing that boundary but positive otherwise. Therefore, if a bubble is crossing the exit between times $t_{1}$ and $t_{2}$, the exact volume of the bubble, $V_{\text {bubble, }}$, can be computed as

$$
V_{\text {bubble }}=\int_{t_{1}}^{t_{2}} Q_{\text {gas }}(t) d t
$$

With the above function, we have plotted in Fig. 11 the bubble radius assuming a spherical geometry, $R_{\text {bubble }}^{S}$ $=\left(3 \pi V_{\text {bubble }} / 4\right)^{1 / 3}$, as a function of $Q$ for different swirl intensities for case 1 in the parameter region where monodisperse bubbles are obtained. Note that, as expected, in all cases the bubble size increases with $Q$. When there is no swirl, the smallest bubble radius obtained for case 1 is about 0.45 times the radius of the tube, while for $S=1.2$ bubbles of 0.14 times $R$ are obtained. In the figure, only two data points
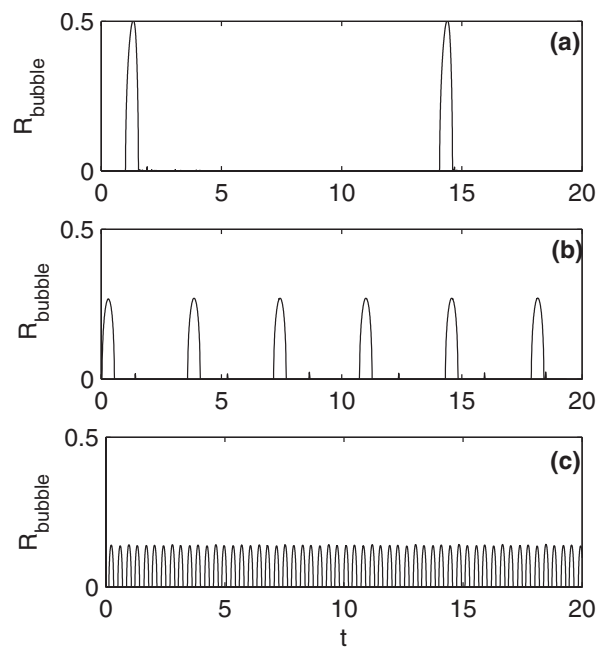

FIG. 10. $R_{\text {bubble }}$ as a function of time for case $1, Q=0.1067$ and three different values of $S$ : (a) $S=0$, (b) $S=0.9$, and (c) $S=1.2$. 

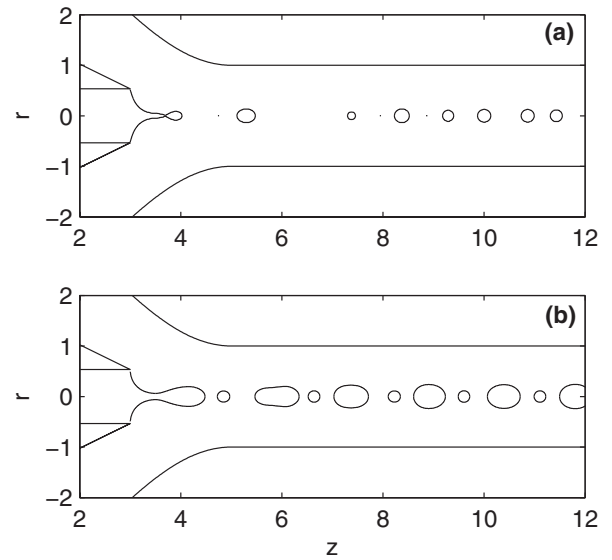

FIG. 11. Radius of the monodisperse spherical bubbles obtained for case 1 as a function of $Q$ for several values of $S$.

for $S=1.2$ are plotted. The reason is the following: increasing swirl leads to smaller bubbles, but the introduction of too much swirl (in this case, $S>1.2$ ) leads to multidisperse bubble breakup. Therefore, as $S$ grows, the $Q$ range for monodisperse bubbles contracts: there is a low- $Q$ range where the gas meniscus recoils into the capillary tube, and there is a high- $Q$ range where satellite drops are produced. In both cases, multidisperse breakup is the result. As $S$ grows, the range reduction is observed not only at the upper limit of the range but also at the lower limit. This is shown in Fig. 12, which depicts a snapshot of the bubble generated for case 1 , $S=1.2$, and two values of $Q$ : (a) $Q=0.00355$ (lower multidisperse range; meniscus recoil) and (b) $Q=0.02844$ (upper multidisperse range). It is worth noting that, for $Q$ $=0.02844$, a perfectly periodic bubbling regime is observed, which periodically alternates the emission of a relatively large bubble with the emission of a much smaller bubble. This behavior is consistent with the dynamical bifurcation described in a similar microbubbling system by Garstecki $e t$ $a .^{24}$

The above results show that the introduction of swirl for case 1 yields a reduction in the size of the monodisperse bubbles produced. It was also seen that the swirl must be
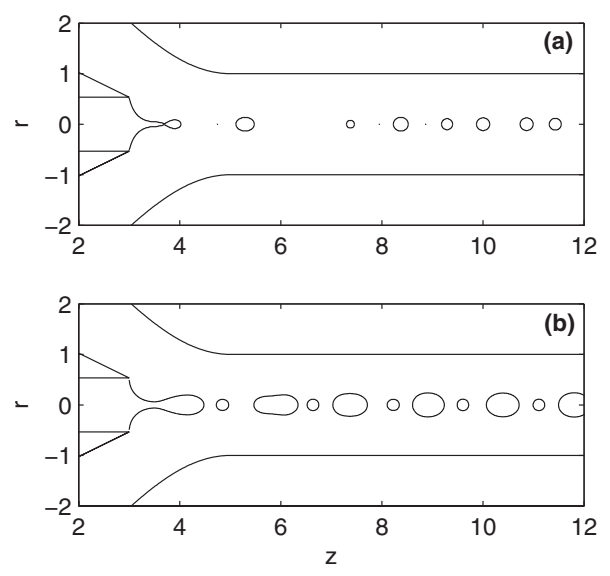

FIG. 12. Instantaneous snapshots showing the nonmonodisperse bubbles generated for case $1, S=1.2$ and two values of $Q$ : (a) $Q=0.00355$ and (b) $Q=0.02844$.
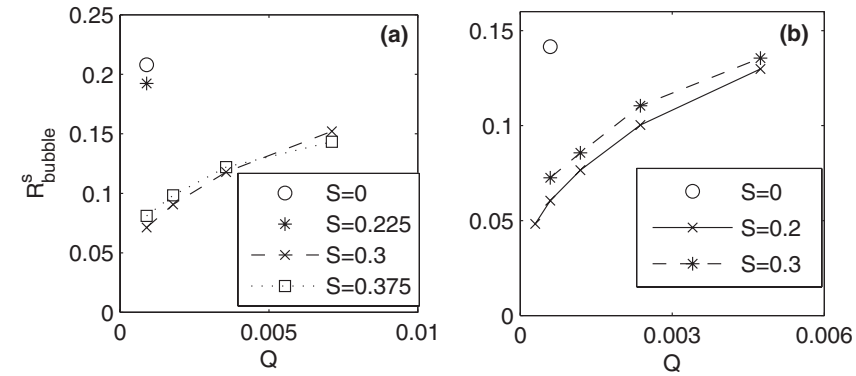

FIG. 13. $R_{\text {bubble }}^{S}$ as a function of $Q$ for several values of $S$ and for (a) case 2 and (b) case 3 .

kept below a certain level, $S_{\max }$, to maximize its beneficial impact. For example, for case $1, S_{\max } \sim 0.9$ since although for $S=1.2$ it is still possible to produce small bubbles, the $Q$ range for monodisperse bubble production is smaller. Let us now explore the effect of swirl in the other four cases considered in this work. Figure 13 shows the parameter range where monodisperse bubbling is observed in cases 2 and 3 . As mentioned in Sec. IV A, when there is no swirl $(S=0)$, it is possible to obtain smaller bubbles by increasing the liquid flow rate (for a given geometrical configuration). In effect, the figures show that one is able to get bubbles with $R_{\text {bubble }}^{S}$ $\sim 0.21$ for case 2 and with $R_{\text {bubble }}^{S} \sim 0.12$ for case 3 in the absence of swirl. However, the main problem for these two cases when swirl is absent is that the production of monodisperse bubbles is restricted to very small values of $Q$. Fortunately, as can be gathered from the figure, the introduction of swirl not only helps get smaller monodisperse bubbles but also get very small ones with a dramatically increased range of $Q$. This means that, for a fixed value of $Q$, the introduction of a suitable swirl level allows the frequency of production to be greatly increased. Note that for case 2 we present results just up to $S=0.375$, since the best results are obtained with $S=0.3$ (values larger than $S=0.375$ yield a further decrease in the $Q$ range for the production of monodisperse bubbles). For case 3, the best results are reached for $S=0.2$. As in case 1, in cases 2 and 3 the improvement in the bubble generation is related to the stabilization of the gas-liquid meniscus due to the swirl. This stabilization allows, if the swirl level lies within a certain range, the bubble generation to change from a meniscus-oscillating (global dripping) regime to a jetting regime. Here, we are not stating that the observed short jet is convectively unstable: indeed, we verified using well established linear stability analysis models ${ }^{26}$ that the short gas ligament exhibited in the cases explored here is absolutely unstable, which explains its shortness. The perturbations that could theoretically proceed upstream due to the absolutely unstable nature of that gas ligament seem, however, blocked by the rapid increase in its radius in the upstream direction from the pinching region. For example, Fig. 14 shows the generation of monodisperse bubbles for case 2 , $Q=0.00089$ and $S=0.3$. One observes that small monodisperse bubbles are periodically emitted from a short but steady gas-liquid meniscus. The strong suction effect around the axis, which provides the acute stabilizing effect just described, may even produce the intriguing meniscus topology which is illustrated in three dimensions in Fig. 14(e), where 
(a)

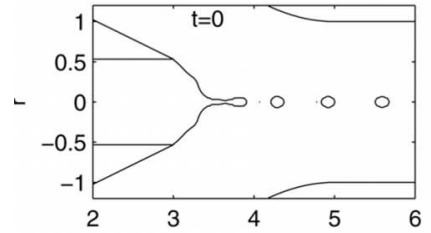

(c)
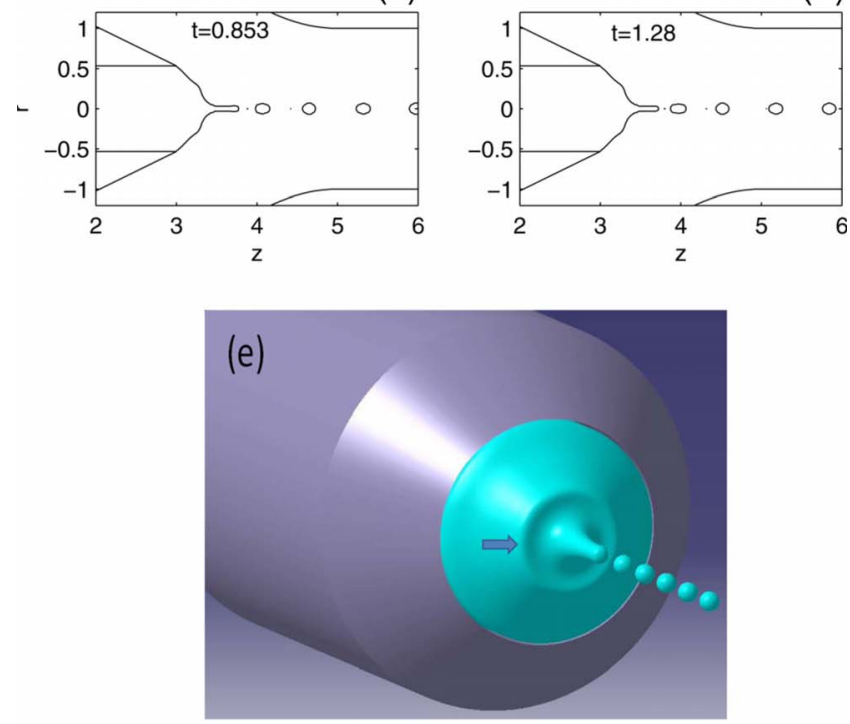

FIG. 14. (Color online) (a)-(d) A sequence of snapshots showing bubble generation for case $2, Q=0.00089$ and $S=0.3$. Note the steadiness of the capillary meniscus. (e) The intriguing, steady circular ripple formed in the middle of the meniscus surface (see arrow), which defines a steady nipple from which the gas ligament and the bubbles issue.

a static axisymmetric "ripple" delimits the conspicuous stable nipple from whose tip the microbubbles are ejected. A deeper study of the details of this flow structure is currently in progress, but we may report in advance that it is a stable structure which is recovered after the introduction of large perturbations.

To conclude, we present in Fig. $15 R_{\text {bubble }}^{S}$ as a function of $Q$ for all cases 1-5 studied in this work. Some remarks about this plot are required. First, in general it can be seen that the introduction of swirl provides a considerable improvement in the generation of monodisperse microbubbles. Second, one observes that the introduction of swirl brings the bubble size, for a given $Q$, right into agreement with the scaling law proposed by Gañán-Calvo ${ }^{21}$ with remarkable accuracy and without resorting to any scaling argument involving the swirl. This apparent paradox can be explained on the same physical basis as that used before: ${ }^{21}$ in the region where bubbles are formed, the dominant dynamical effects are the local and the convective accelerations driven by the applied pressure gradient. As long as $S \lesssim 1$, the pressure gradient in the axial direction is dominant and proportional to $\rho_{l} W^{2} / R$, while that in the radial direction is at most of the same order as the former. Thus, the scaling arguments made by Gañán-Calvo $^{21}$ are perfectly valid here. The actual fundamental role of the swirl when $S \lesssim 1$ is to provide a lowpressure region at the axis (local suction effect) which stabi-

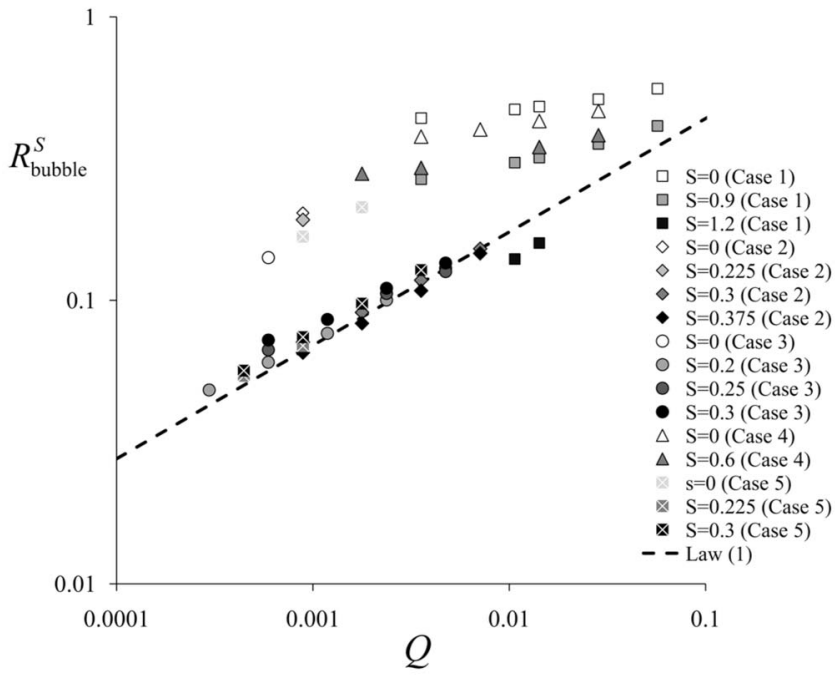

FIG. 15. $R_{\text {bubble }}^{S}$ as a function of $Q$ for several values of $S$.

lizes the tapering meniscus from which the gas bubbles issue. In other words, the role of swirl is subdominant but provides the route to a stable tapering topology, the only one producing the smallest bubbles at the highest possible frequency. One may consistently observe that, in those cases where $S$ surpasses the unity, the bubble sizes fall slightly below the prediction with subdominant swirl (see the two points for $S=1.2$, case 1 , in Fig. 15). In this case, the radial pressure gradients provided by the swirl surpass $\rho_{l} W^{2} / R$ (the azimuthal velocities are about $20 \%$ larger than the axial ones) and the effect of swirl becomes dominant, producing a deviation from the original scaling law. ${ }^{21}$

In summary, our results indicate that

(i) the introduction of swirl induces the stabilization of the tapering meniscus with a steady neck or short gas ligament from which the bubbles issue;

(ii) once the gas ligament is stabilized, the bubble formation follows the same physics as discussed by Gañán-Calvo $^{21}$ as long as $S \lesssim 1$, lending support to its universality; and

(iii) accurate, full numerical simulation seems a perfectly valid alternative to experiments in this flow regime of laminar, moderate-to-high Reynolds with free surfaces. $^{39-42}$

As a final remark, potential nonaxisymmetric instabilities of the gas jet become unfavored or inhibited by the swirl action of the coflowing liquid. ${ }^{43}$ This lends support to choice of purely axisymmetric simulation in this study.

\section{ACKNOWLEDGMENTS}

The many suggestions of Pascual Riesco-Chueca during this work are highly appreciated. The help and encouragement of José M. López-Herrera is also much appreciated. The support from the Ministry of Science and Education (Spain) and from the Andalusian Government through Grants Nos. DPI2007-63559 and Excelencia/TEP-1190, respectively, are gratefully acknowledged. A.M.G.-C. wishes to 
warmly express his gratitude to Piotr Garstecki for many insightful ideas and encouragement and to Juan Fajardo Lopez-Cuervo, MD, for his encouragement, collaboration, and vision on the use of microbubbles in biomedical applications.

${ }^{1}$ P. N. T. Wells, "Physics and engineering: Milestones in medicine," Med. Eng. Phys. 23, 147 (2001).

${ }^{2}$ E. Stride and N. Saffari, "Microbubble ultrasound contrast agents: A review,” Proc. Inst. Mech. Eng., Part H: J. Eng. Med. 217, 429 (2003).

${ }^{3}$ E. C. Unger, T. Porter, W. Culp, R. Labell, T. Matsunaga, and R. Zutshi, "Therapeutic applications of lipid-coated microbubbles," Adv. Drug Delivery Rev. 56, 1291 (2004).

${ }^{4}$ B. J. Schmidt, I. Sousa, A. A. van Beek, and M. R. Böhmer, "Adhesion and ultrasound-induced delivery from monodisperse microbubbles in a parallel plate flow cell," J. Controlled Release 131, 19 (2008).

5 J. G. Riess, "Perfluorocarbon-based oxygen delivery," Artif. Cells Blood Substit Immobil Biotechnol. 34, 567 (2006).

${ }^{6}$ F. Gerber, G. Waton, M. P. Krafft, and T. F. Vandamme, "Long lived microbubbles for oxygen delivery," Artif. Cells Blood Substit Immobil Biotechnol. 35, 119 (2007).

${ }^{7}$ M. A. Borden and M. L. Longo, "Dissolution behavior of lipid monolayercoated, air-filled microbubbles: Effect of lipid hydrophobic chain length," Langmuir 18, 9225 (2002).

${ }^{8}$ M. Barak and Y. Katz, "Microbubbles: Pathophysiology and clinical implications," Chest 128, 2918 (2005).

${ }^{9}$ J. A. Feshitan, C. C. Chen, J. J. Kwan, and M. A. Borden, "Microbubble size isolation by differential centrifugation," J. Colloid Interface Sci. 329, 316 (2009).

${ }^{10}$ U. Farook, E. Stride, M. J. Edirisinghe, and R. Moaleji, "Microbubbling by co-axial electrohydrodynamic atomization," Med. Biol. Eng. Comput. 45, 781 (2007).

${ }^{11}$ Y. Li, J. Li, H. B. Zhang, and Y. S. Su, "Microbubble suspensions prepared via electrohydrodynamic jetting process," BMEI, International Conference on BioMedical Engineering and Informatics, 2008, Vol. 2, pp. 445-449.

${ }^{12}$ A. M. Gañán-Calvo and J. M. Gordillo, "Perfectly monodisperse microbubbling by capillary flow focusing," Phys. Rev. Lett. 87, 274501 (2001).

${ }^{13}$ P. Garstecki, I. Gitlin, W. DiLuzio, E. Kumacheva, H. A. Stone, and G. M. Whitesides, "Formation of monodisperse bubbles in microfluidic flowfocusing device," Appl. Phys. Lett. 85, 2649 (2004).

${ }^{14}$ P. Garstecki, A. M. Gañán-Calvo, and G. M. Whitesides, "Formation of bubbles and droplets in microfluidic systems," Bull. Pol. Acad. Sci.: Tech. Sci. 530, 1 (2005).

${ }^{15}$ P. Garstecki, M. J. Fuerstman, H. A. Stone, and G. M. Whitesides, "Formation of droplets and bubbles in a microfluidic t-junction-scaling and mechanism of break-up," Lab Chip 6, 437 (2006).

${ }^{16}$ A. M. Gañán-Calvo, Patent No. WO2005044434 (2005).

${ }^{17}$ K. P. Pancholi, U. Farook, R. Moaleji, E. Stride, and M. J. Edirisinghe, "Novel methods for preparing phospholipid coated microbubbles," Eur. Biophys. J. 37, 515 (2008).

${ }^{18}$ S. Takeuchi, P. Garstecki, D. B. Weibel, and G. M. Whitesides, "An axisymmetric flow-focusing microfluidic device," Adv. Mater. (Weinheim, Ger.) 17, 1067 (2005).

${ }^{19}$ A. M. Gañán-Calvo, "Generation of steady liquid microthreads and micron-sized monodisperse sprays in gas streams," Phys. Rev. Lett. 80, 285 (1998).

${ }^{20}$ J. Eggers and E. Villermaux, "Physics of liquid jets," Rep. Prog. Phys. 71, 036601 (2008)

${ }^{21}$ A. M. Gañán-Calvo, "Perfectly monodisperse microbubbling by capillary flow focusing: An alternate physical description and universal scaling," Phys. Rev. E 69, 027301 (2004).

${ }^{22}$ G. I. Barenblatt, Scaling, Self-similarity, and Intermediate Asymptotics (Cambridge University Press, Cambridge, 1996).

${ }^{23}$ P. Garstecki, H. A. Stone, and G. M. Whitesides, "Mechanism for flowrate controlled breakup in confined geometries: A route to monodisperse emulsions," Phys. Rev. Lett. 94, 164501 (2005).

${ }^{24}$ P. Garstecki, M. J. Fuerstman, and G. M. Whitesides, "Nonlinear dynamics of a flow-focusing bubble generator: An inverted dripping faucet," Phys. Rev. Lett. 94, 234502 (2005).

${ }^{25}$ L. Yobas, S. Martens, W.-L. Onga, and N. Ranganathan, "Highperformance flow-focusing geometry for spontaneous generation of monodispersed droplets," Lab Chip 6, 1073 (2006).

${ }^{26}$ A. M. Gañán-Calvo, M. A. Herrada, and P. Garstecki, "Bubbling in unbounded coflowing liquids," Phys. Rev. Lett. 96, 124504 (2006).

${ }^{27}$ M. A. Herrada, A. M. Gañán-Calvo, A. Ojeda-Monge, B. Bluth, and P. Riesco-Chueca, "Liquid flow focused by a gas: Jetting, dripping, and recirculation," Phys. Rev. E 78, 036323 (2008).

${ }^{28}$ D. B. Kothe and W. J. Rider, "A comparison of interface tracking methods," in 12th AIAA CFD Conference (1995).

${ }^{29}$ S. O. Unverdi and G. Tryggvason, "A front-tracking method for viscous, incompressible, multi-fluid flows," J. Comput. Phys. 100, 25 (1992).

${ }^{30}$ D. J. E. Harvie and D. F. Fletcher, "A new volume of fluid advection algorithm: The stream scheme," J. Comput. Phys. 162, 1 (2000).

${ }^{31}$ E. Aulisa, S. Manservisi, and R. Scardovelli, "A mixed markers and volume-of-fluid method for the reconstruction and advection of interfaces in two-phase and free-boundary flows," J. Comput. Phys. 188, 611 (2003).

${ }^{32} \mathrm{~S}$. Tanguy and A. Berlemont, "Application of a level set method for simulation of droplet collisions," Int. J. Multiphase Flow 31, 1015 (2005).

${ }^{33}$ C. W. Hirt and B. D. Nichols, "Volume of fluid (vof) method for the dynamics of free boundaries," J. Comput. Phys. 39, 201 (1981).

${ }^{34} \mathrm{P}$. Heinrich, "Nonlinear numerical model of landslide-generated water waves," Int. J. Eng. Fluid Mech. 4, 403 (1991).

${ }^{35}$ A. Tomiyama, A. Sou, H. Minagawa, and T. Sakaguchi, "Numerical analysis of a single bubble by VoF method," JSME Int. J., Ser. B 36, 51 (1993).

${ }^{36}$ B. Lafaurie, C. Nardone, R. Scardovelli, S. Zaleski, and G. Zanetti, "Modelling merging and fragmentation in multiphase flows with surfer," J. Comput. Phys. 113, 134 (1994).

${ }^{37}$ B. Van Leer, "Toward the ultimate conservative difference scheme. IV. A second order sequel to Godunov's method," J. Comput. Phys. 32, 101 (1979).

${ }^{38}$ R. I. Issa, "Solution of implicitly discretized fluid flow equations by operator splitting," J. Comput. Phys. 62, 40 (1986).

${ }^{39}$ E. D. Wilkes, S. D. Phillips, and O. A. Basaran, "Computational and experimental analysis of dynamics of drop formation," Phys. Fluids 11, 3577 (1999).

${ }^{40}$ A. U. Chen, P. K. Notz, and O. A. Basaran, "Computational and experimental analysis of pinch-off and scaling," Phys. Rev. Lett. 88, 174501 (2002).

${ }^{41}$ R. Suryo and O. A. Basaran, "Tip streaming from a liquid drop forming from a tube in a co-flowing outer fluid," Phys. Fluids 18, 082102 (2006).

${ }^{42}$ H. J. Subramani, H. K. Yeoh, R. Suryo, Q. Xu, B. Ambravaneswaran, and O. A. Basaran, "Simplicity and complexity in a dripping faucet," Phys. Fluids 18, 032106 (2006)

${ }^{43}$ S. P. Lin, Breakup of Liquid Sheets and Jets (Cambridge University Press, Cambridge, 2003). 